\title{
II. Visual diffusivity
}

\section{Herbert E. Ives}

To cite this article: Herbert E. Ives (1917) II. Visual diffusivity , Philosophical Magazine Series 6, 33:193, 18-33, DOI: $10.1080 / 14786440108635613$

To link to this article: http://dx.doi.org/10.1080/14786440108635613

$$
\text { 册 Published online: } 08 \text { Apr } 2009 .
$$

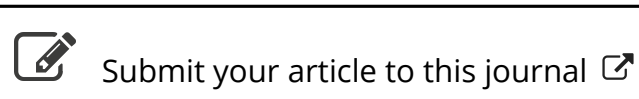

LII Article views: 3

Q View related articles ¿

4 Citing articles: 2 View citing articles 


\section{Concluding Remaris.}

In surveying the results so far obtained, it must be borne in mind that the present research is necessarily only of a preliminary nature, owing to the restrictions imposed by the optical means employed. Nevertheless, most valuable information and experience have been gained, which will prove of great help in planning the course along which future research will have to be conducted. Thus the division of lines into classes might profitably be carried still further by taking into account the various types of enhanced lines, to which I drew attention at the last meeting of the International Union for Solar Research*. This, combined with an extension of the spectral region examined in the ultra-violet and towards the longer wave-lengths, would, no doubt, bring in many more line groups, and perhaps ultimately lead to the discovery of the complete structure of the spectrum. The grouping of apparently connected lines which has, so far, been accomplished as a result of this investigation, may be regarded as a first step in that direction.

In conclusion, I desire to express my gratitude to Sir Ernest Rutherford for the most generous hospitality which he is extending to me in his laboratory, and to thank him for the kind interest he is taking in the work.

My thanks are also due to Professor Beattie, in whose laboratory workshop many of the apparatus used in this research have been constructed.

Manchester, Nov. 2, 1916.

1. Introduction.

II. Visual Diffusivity. By Herbert E. IVES $\dagger$.

2. Application of the Idea of Diffusivity to Instantaneous Light Sources.

3. Qualitative Observations on Retarded Images and After-Images.

4. Measurements.

5. Discussion.

\section{Introduction.}

T $N$ two provious papers by the writer and Mr. E. F. 1 Kingsbury $\ddagger$ on "The Theory of the Flicker Photometer," the idea has been developed that the transmission of impressions from the retina to the brain is in accordance

* Bonn, 1913.

+ Communicated by the Author.

+ Ives and Kingsbury: "The Theory of the Flicker Photometer." I. \& II. Phil. Mag., Nov. 1914, p. 708; April 1916, p. 290. 
with the physical laws of conduction. The chief experimentally established phenomena of the flicker photometer find explanation in the assumption that the stimulus is transmitted through a layer of matter having a coefficient of diffusivity which is different for different colours and varies with the intensity of the stimulus. In accordance with our previous work, the diffusivity for coloured light is a rectilinear function of the logarithm of the stimulus intensity. For colourless or rod vision the diffusity is constant. These relations are exhibited diagrammatically in fig. 1.

Fig. 1.

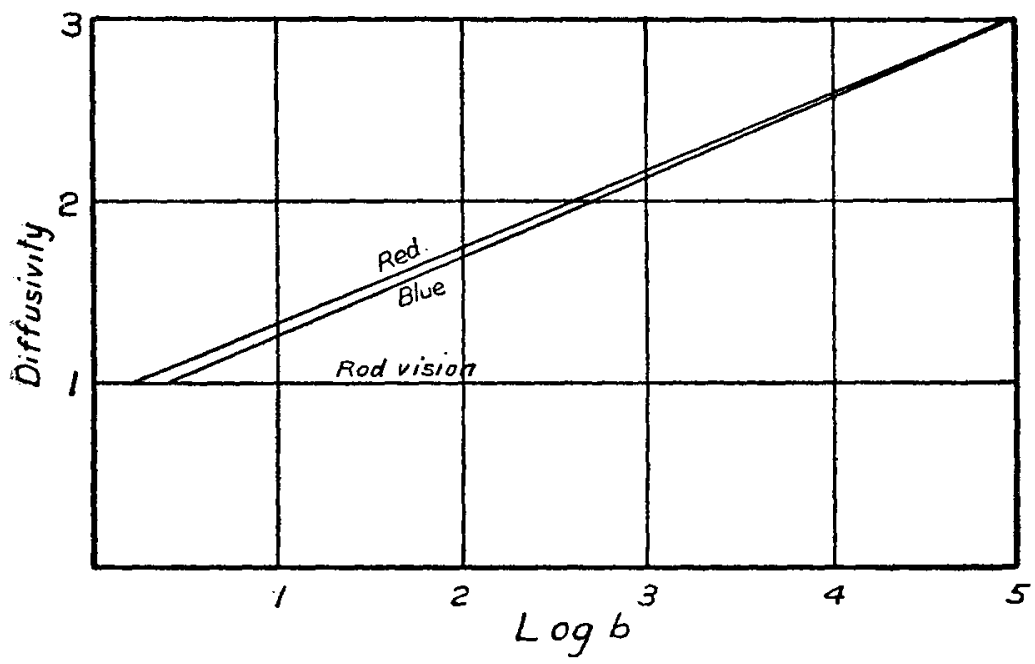

Diagrammatic representation of brightness-diffusivity relation for red, blue, and rod vision.

This conception of the visual process has not only served to explain previously known facts in the realm of intermittent vision, but has indicated others not heretofore observed. The phenomena of flicker are, however, of such a complex character that, satisfactory as this hypothesis has been, it may have appeared to some to be insufficiently supported so long as it played no part in explaining other simpler phenomena.

The present paper deals with the visual parallel to one of the simplest cases of heat conduction, in which the diffusivity of the conducting layer figures, namely that of instantaneous 
heat sources. This, in the case of light, is of course the case of single brief exposures. It is shown below that, in accordance with our theory, a difference of diffusivity should result in a difference in the time of perception after exposure. This predicted difference has been found both in the case of light of one colour but different intensity and in the case of lights of different colour and the same intensity. In addition, the positive after-image has been indicated to be simply the rod-vision impression delayed in transmission by the low diffusivity peculiar to this kind of vision. Both the new phenomena of differential rate of transmission for photopic stimuli and the relation of the colourless after-image to the photopic images have been subjected to quantitative measurement with results supporting the theory.

\section{Application of the Idea of Diffusivity to Instantaneous Light. Sources.}

It is shown in treatises on heat conduction that the temperature $V$ of a conducting slab of diffusivity $K$, at a depth $X$, at a time $t$ after the instantaneous application to the surface of a quantity of heat $Q$ is

$$
\frac{\mathrm{Q}}{2 \sqrt{\overline{\mathrm{K}} \pi t}} e^{-\frac{\mathrm{X}^{2}}{4 \mathrm{~K} t}} \cdot \ldots . . . \cdot .
$$

Now the time at which this is a maximum may be found by differentiating (1) with respect to $t$. This gives

$$
t_{m}=\frac{\mathrm{X}^{2}}{2 \mathrm{~K}}, \cdot \cdot \cdot \cdot \cdot \cdot \cdot \cdot .
$$

or the interval between reception and perception of a stimulus is inversely as the diffusivity.

The measurement of this interval is obviously not feasible, but the difference between the intervals for two stimuli is measurable. Several special cases may be treated :-

1st Case.

One colour, two intensities, $I_{1}$ and $I_{2} ; I_{2}>I_{1}$.

$$
\begin{aligned}
t_{1} & =\frac{1}{a \log \mathrm{I}_{1}+b}, \quad . \quad . \quad . \quad . \quad . \quad . \\
t_{2} & =\frac{1}{a \log \mathrm{I}_{2}+b}, \quad . \quad . \quad . \quad . \quad . \quad . \\
t_{1}-t_{2} & =\frac{1}{a \log \mathrm{I}_{1}+b}-\frac{1}{a \log \mathrm{I}_{2}+b} . . .
\end{aligned}
$$


This case is plotted in fig. 2, using the data of fig. 1, taking $\mathrm{I}_{2}=10 \mathrm{I}_{1}$. The less intense image should be seen later than the more intense by an interval which should increase with decreasing intensity. If the observation is by

Fig. 2.

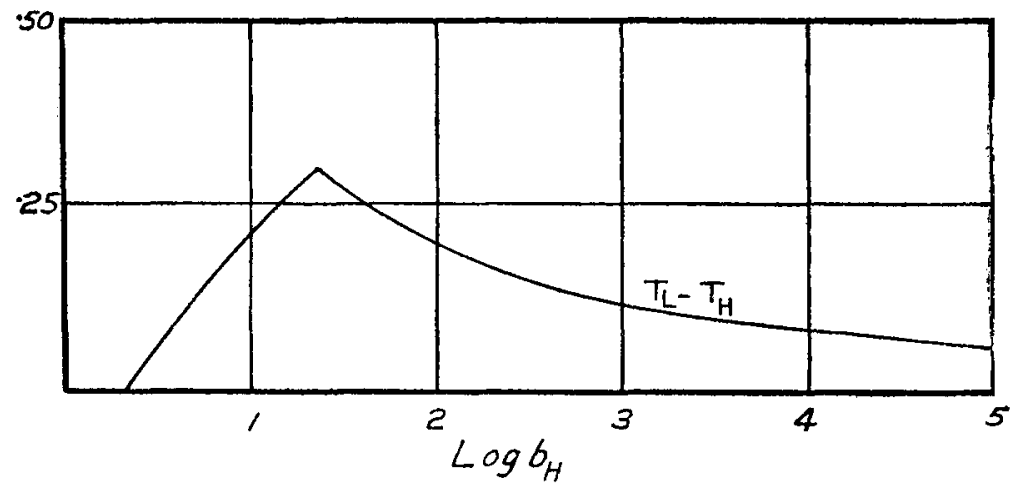

Time-interval between a high intensity and a low intensity image (differing ten times in brightness), as calculated from the data of fig. 1.

blue light, which goes over into rod vision at the lowest intensities, this interval again decreases, becoming zero when the diffusivity is constant and the same for both images. This is the case represented.

2nd Case.

Two colours, red and blue, of the same intensity.

$$
\begin{aligned}
& t_{\mathrm{R}}=\frac{1}{a \log \mathrm{I}+b}, \quad \cdot \quad \cdot \quad \cdot \quad \cdot \quad . \quad . \\
& t_{\mathrm{B}}=\frac{1}{c \log I+d}, \quad . \quad . \quad . \quad . \quad . \quad . . \\
& t_{\mathrm{B}}-t_{\mathrm{R}}=\frac{\mathrm{I}}{c \log \mathrm{I}+d}-\frac{1}{a \log \mathrm{I}+b} . . .
\end{aligned}
$$

This case is plotted in fig. 3 , again using the data of fig. 1 . For all intensitios shown on the plot the blue image will be seen later than the red. At high intensities the interval will be smaller than at low. At very low intensities, where the 
blue image merges into the rod image the red and blue will again approach each other.

Fig. 3.

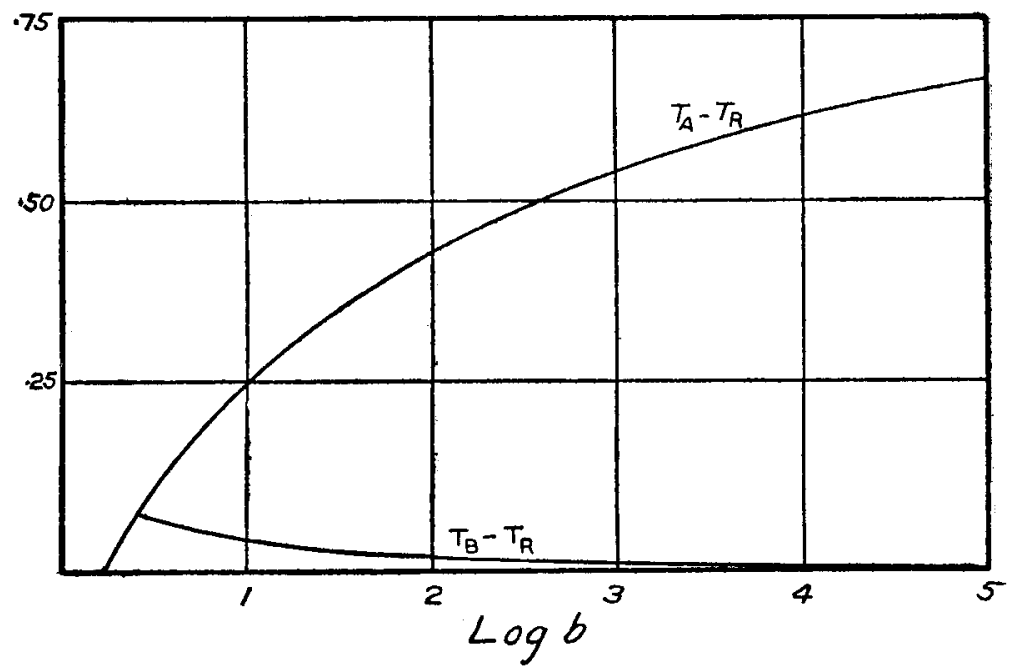

Time-interval between blue and red, and between red and after-images, calculated from data of fig. 1 .

3rd Case.

A coloured (photopic) image, and a colourless (scotopic) image.

It has been a debated question whether the colourless rod image is actually present at high intensities. On the assumption that it is, we have the condition

$$
\begin{aligned}
t_{\mathrm{C}} & =\frac{1}{a \log \mathrm{I}+b}, \quad . \quad . \quad . \quad . \\
t_{\mathrm{A}} & =\frac{1}{g}, \quad . \quad . \quad . \quad . \quad . \quad . \\
t_{\mathrm{A}}-t_{\mathrm{C}} & =\frac{1}{g}-\frac{1}{a \log \mathrm{I}+b} .
\end{aligned}
$$

If we call the rod image the after-image, it should lag behind the photopic image by an interval which increases with the intensity in the manner shown in fig. 3 . If the interval is measured after a blue image it becomes zero. 
where this image merges with the rod image. If measured after a red image it becomes equal to the blue-red interval at the same point.

\section{Qualitative Observations on Retarded Images and After-Images.}

(a) Apparatus.

The apparatus, which was designed so as to permit of measurement of the time-interval between two impressions, is shown diagrammatically in fig. 4 . It consists essentially

Fig. 4.

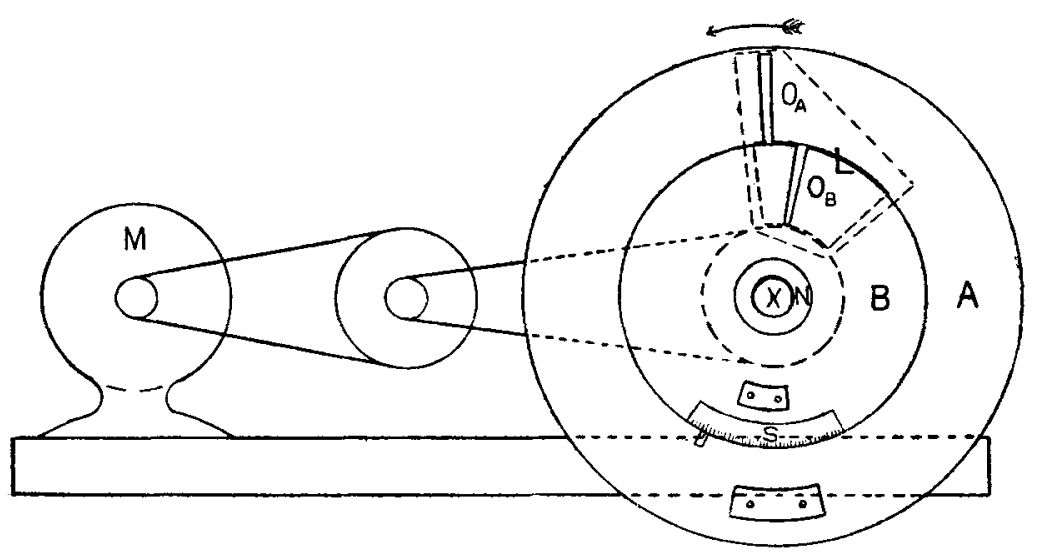

Diagram of apparatus for studying interval between perception of images of different brightness and colour.

of two superposed circular disks of sheet metal, A and B, one $22 \mathrm{~cm}$. the other $36 \mathrm{~cm}$. diameter, both mounted on the same axis, $X$, and locked in any relative angular relation by the nut $N$. In each disk is narrow radial slot, $O$, of five degrees opening, the relative positions of these slots being variable from coincidence of direction up to about sixty degrees divergence, as read on the scale $S$. On the back of the larger disk is mounted a piece of flashed opal glass which is illuminated by the light source behind and is the actual bright surface observed. Grooved holders are provided over $\mathrm{O}_{\mathrm{A}}$ and $\mathrm{O}_{\mathrm{B}}$ for carrying coloured or other glasses, and the small streak of light between the disks at $\mathrm{L}$ is covered by strips of electric wiring tape. The disks are rotated slowly by an electric motor with a multiple reduction pulley system by which speeds of one revolution per second and less are 
obtainable. Speeds are measured by an electric tachometer. An elaborate screening system is provided so that no light gets around the edge of the disks, and observations are made at one metre distance in a darkened chamber.

The principle which the apparatus is planned to utilize is a familiar one. If the slot $\mathrm{O}_{A}$ be imagined covered with a glass to reduce its intensity, or with a coloured glass of a hue more towards the blue than that of $\mathrm{O}_{\mathrm{B}}$, the theory just developed predicts that on rotating the disks in the counterclockwise direction, with the slots exactly in line, they will be seen not in line, but the slot $\mathrm{O}_{\mathrm{A}}$ will appear to lag behind or be displaced. If this occurs, then it will be possible to move the disk $B$ in the clockwise direction until on rotation the two slots again appear in line. Knowing the angle by which the slots have been turned with respect to each other, and the angular velocity of the disks, it is easy to calculate the interval of time by which one impression is delayed behind the other.

Before attempting quantitative measurement of these phenomena they were studied in detail qualitatively, and a description of these observations follows :-

(b) Light and dark stimuli of the same colour.

The slot $O_{A}$ is covered by a neutral tint glass, the two slots being in line, and the disks are slowly rotated. At a fairly low intensity of illumination (illumination and other

Fig. 5.

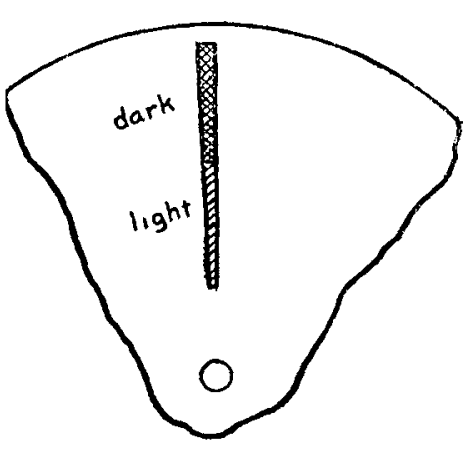

a

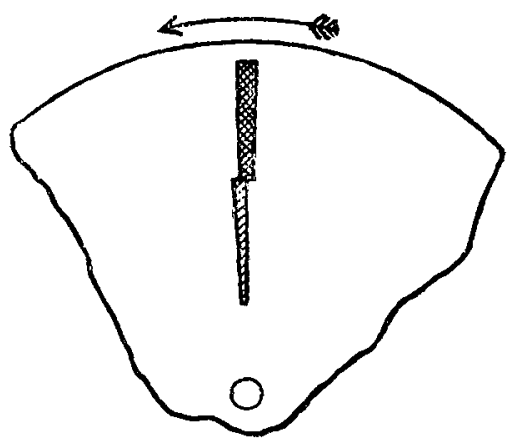

b

Appearance of adjacent dark and light apertures, or blue and red apertures: $a$, stationary; $b$, in motion.

constants are given in the description of the measurements) the dark slot appears several degrees behind the other, presenting the appearance shown in fig. $5 b$. At high 
intensities this phenomenon is less marked, as it is again at very low ones. The prediction of theory is confirmed.

\section{(c) Red and blue light.}

The slot $\mathrm{O}_{\mathrm{A}}$ is covered by a good blue glass, which transmits no red, and the slot $\mathrm{O}_{B}$ by a red signal glass. On rotation the blue image lags behind by several degrees, presenting the appearance already depicted by fig. $5 b$, substiruting the difference of colour for the difference of intensity which the figure indicated when referring to the last section.

This lagging of one colour behind the other is a very striking phenomenon, well suited to demonstration to a small audience. The effect is most extraordinary if the disk, instead of being rotated, is rapidly oscillated through five or ten degrees, when the blue image may be made to vibrate entirely out of phase with the red, appearing as though attached to it by a cord. It is not an effect that has to be glimpsed after painful preparation by a trained eye, but an optical delusion of most convincing verisimilitude. On its being exhibited without comment to the mechanic who made the apparatus, he immediately assumed that he had been called in to correct a mechanical slip between the disks :

At the very high intensity obtained by placing a carbon are lamp about 35 centimetres behind the flashed opal glass of the disk, the lag of blue behind red is practically absent; it was not found possible with the intensities at our disposal to demonstrate a reversal of the red and blue positions, such as theory indicates for some excessively high intensity, beyond the crossing point of the red and blue diffusivity lines of fig. 1.

(d) Purple light.

If the various colour impressions are transmitted by entirely independent channels, it would follow that a narrow line of a mixed colour such as purple ought to be resolved irto its constituents when passed across the field of vision. Dr. Karrer informs me that he has observed a moving bright light spread out into a spectrum in a way which would meet with explanation in this way. I was not able, however, to obtain a clear cut and unmistakable resolution of any purple I tried with this apparatus. This agrees with the conclusion drawn from the previous work with the flicker photometer with unequal exposures, namely, that the difference in the diffusivities peculiar to the two colours is much reduced when they are simultaneously transmitted by the same retinal area, although it is still present to some degree, as 
the behaviour of the flicker photometer shows. This apparent mutual action does not, however, appear to occur between the photopic and the after-images. The latter behave as though due to an entirely separate mechanism.

(e) After-images.

The most characteristic feature of the positive after-image, if it actually is not a recurrent impression, but the slowly transmitted impression through the rods, as the present theory would indicate, would be that the interval between it and the photopic image would be variable with the intensity. With decreasing intensity the after-image should move in towards the blue image, and at very low intensities should merge into it.

Another characteristic of this after-image would be that, in accordance with the well-established wave-length sensibility of the eye for scotopic vision, the red end of the spectrum is practically inoperative-that is, there should be no after-image of a red stimulus.

These phenomena are all exhibited by providing the slots with red, green, and blue glasses, in the manner indicated in fig. $6 a$. On rotation the three colours are shifted out of line, in accordance with the subject-matter of the previous sections, and at some distance behind the green and blue

Fig. 6.
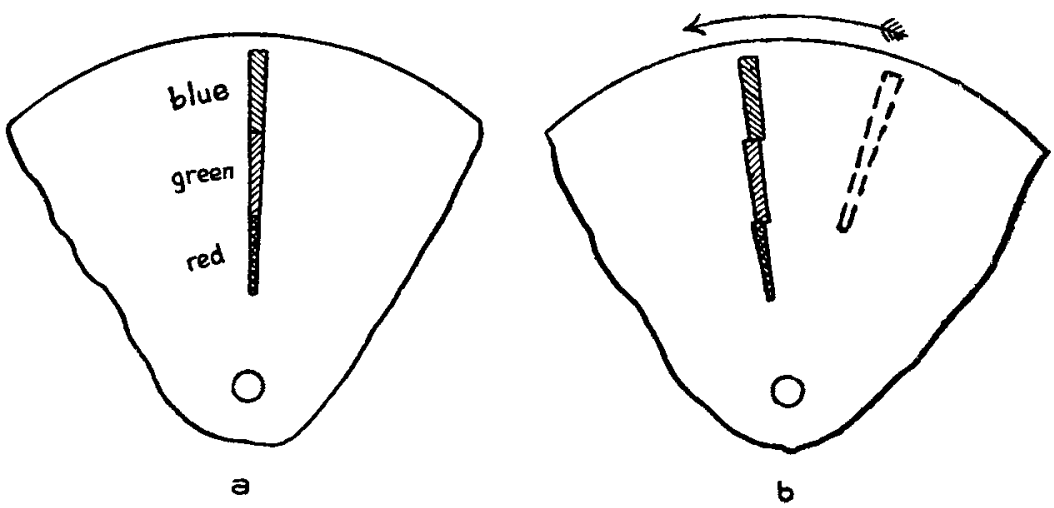

Appearance of red, green, and blue apertures lying in straight line: $a$, stationary; $b$, in motion.

images-but not behind the red-is the colourless after-image, the after-images of the two colours appearing in the same straight line, as they should from the theory. The appearance at this stage is as shown in fig. $6 b$. On decreasing the 
intensity the after-image moves in closer ; at rather lower intensity the after-image appears merely as a grey tail to the green and blue images; while at the very lowest intensities these latter become single grey images.

An observation worthy of record, though probably not new, is that the after-image when only slightly separated from the blue primary image is distinctly the brighter of the two.

\section{Measurements.}

(a) Instrumeutal conditions and constants.

For the greater part of the measurements the rotating sector disk was illuminated by a tungsten lamp of approximately 100 candle-power, mounted on an eight metre photometer track to permit of a wide variation of illumination by variation of distance. The brightness of the opal glass without any colour screen was one candle per square centimetre at the point marked on the arbitrary logarithmic scale of fig. 7. The photometric transmission

Fig. 7 .

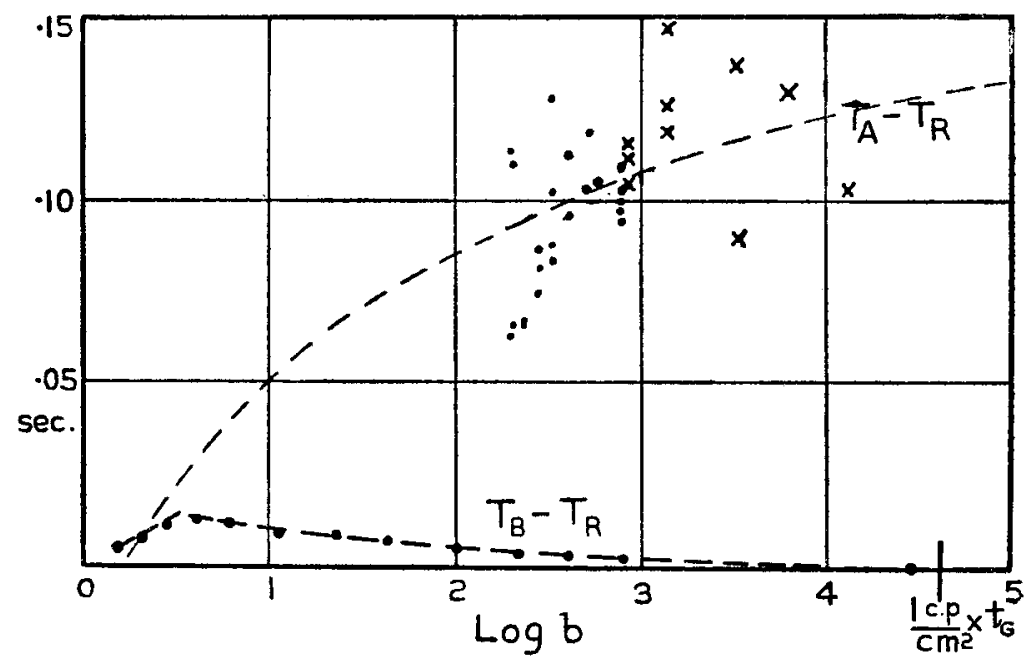

Experimental results. Time-intervals between blue and red image perceptions, and between after and red image perceptions.

of the red and blue glasses used over the opal glass was nearly the same, probably not far from five per cent. for the high brightness conditions. The relative brightness of 
course varied greatly with the illumination, due to the Purkinje phenomenon.

It is of considerable importance that the eye be properly fixated at the centre of rotation of the disks. The best means found for insuring this was to faintly illuminate the chalkmarked centre of the axle by a beam of light from a distant light and mirror.

The observations were made by the chief observer in a carefully darkened booth. Speeds of rotation were regulated by an adjustable resistance in series with the disk motor. Speeds were read and lamp positions changed by an assistant *.

In this work the artificial pupil heretofore consistently used was omitted because it was found that, owing to the chromatic aberration of the eye, slight unavoidable eyemovements cause relative movements of the red and blue images similar in appearance to those due to the diffusivity differences, and bence likely to be confused with them in measurement.

(b) Measurements on red and blue light.

No measurements were made on two lights of different intensity (case 1 above), as the case of two colours practically includes it, and is more striking and easily measurable, because of the approximate equality of brightness which may be arranged for in the two colours under observation.

A series of values of the time-interval between the red and blue light used, for different illuminations, is shown in fig. 7. In making this series the angle between $O_{A}$ and $O_{B}$ was one degree at the higher intensities and two degrees at the lower. The employment of larger angles and hence higher speeds, which would at first thought appear preferable, was actually found to be rather less conducive to accuracy because the images begin to spread out and so lose in definiteness of outline.

The predicted behaviour of the two colours with respect to each other is borne out by the measurements.

(c) Measurements on after images.

Measurements on the positive after-image, or slowly transmitted rod image as it is here considered to be, are extremely difficult. Two kinds of difficulty may be mentioned-those of fixation and those of visibility. It is absolutely mecessary, if accurate measurements are to be

* Acknowledgment is due $\mathrm{Mr}$. E. R. Morton for assistance in the experimental work. 
made, that the eye be steadily fixed at the centre of rotation of the disks. If the eye follows the image, as its tendency is, because of the faintness of the phenomenon, the afterimage is shifted forward, by an amount which can entirely vitiate the measurement. The manner in which this shift occurs is rather interesting, as it shows clearly that the eye-ball does not rotate, but keeps its axis parallel as it follows the bright spot on the disk. The after-image thus does not merely move in closer, but appears to stand at an angle to the primary image, and if the eye-movement is rapid enough it may actually lie diagonally across this, as shown in fig. 8 .

Fig. 8.
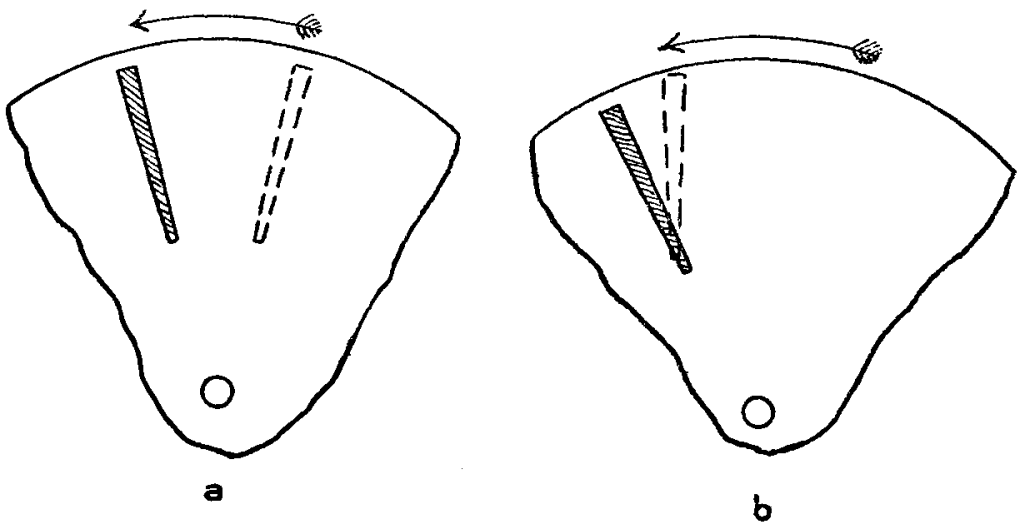

Appearance of after-image (shown dashed); $a$, with proper fixation at centre of rotation: $b$, with improper fixation.

Accurate fixation at the centre of rotation is assured by having this faintly illuminated. With accurate fixation, however, the difficulties due to the low visibility of the image are rather enhanced, for one's attention is distracted from the part of the field in which the after-image appears. From one cause or another the illumination region in whish any measurements at all can be made on the after-image is quite limited. Thus at a rather high illumination the afterimage disappears entirely, as though the rods ceased to function. At an illumination somewhat below this the after-image is fairly bright, and well separated from the primary at moderate speeds. It is here at its best visibility. From here down it becomes less bright, at the same time moving in toward the primary, both changes reducing its visibility. A faint image close to a bright one, whatever 
the nature of the objects, is hard to see, especially when in motion. An increase of speed, which would increase the separation, is no aid because of the spreading out and loss of definition of both images. As a consequence the afterimage practically disappears, especially as a measurable thing, until at very low illuminations the primary (blue) image becomes, as noted, of the same order of brightness as the after-image, when the latter again appears close in, but, because of the low brightness, not easily susceptible of exact measurement.

A further obstacle to measurement is the fact that the eye quickly loses its ability to see the after-image, and must be rested. This does not appear to be at all a matter of adaptation, in particular of dark adaptation, but of time alone. It was found by experience that the eye was best prepared by an interval between observations of fifteen or twenty minutes at least of use in ordinary occupations of reading, \&c., in the normally day-lighted room. Probably under these conditions the rod function is completely inhibited and permitted to recuperate, whereas at the illuminations where both primary and after-image are present this same function is being more rapidly exhausted than its normal rate of recuperation under conditions of pure rod vision.

The measurements obtained are shown in fig. 7. The dots were observations made with the tungsten lamp, as described above, the after-image being brought into coincidence with the red image of the other aperture placed from ten to twenty degrees behind. The crosses represent observations made with an arc lamp with both apertures blue, a condition presenting some advantage at those intensities where the red aperture becomes decidedly brighter in appearance than the blue, due to the Purkinje effect. All observations are, however, reduced to give the interval between red and afterimage, by use of the blue-red interval previously determined for the same conditions. It will be seen that these observations, limited in range and lacking in precision for the reasons given, are in agreement, so far as they go, with the hypotheses presented as to the nature of the after-image. They are not sufficient in range or precision to prove it, and so its chief support must be in the qualitative observations described above.

In an effort to find conditions of easier visibility for the after-image a different form of apparatus was improvised, consisting of two white strips carried on an axis like the hands of a clock. One of these was turned through an angle 
of ien to twenty degrees, and the two then rotated together until the after-image of one appeared in line with the other. The advantage of this apparatus was that the eye could be much more easily kept fixated at the centre of rotation, half of the phenomenon of interest being on each side of the centre. In the apparatus constructed illumination was necessarily from the front, which made it difficult to have a perfectly black background; perhaps substantially better results could be obtained with a similar device arranged for transmitted light, for the improvement in ease of fixation is considerable. However, there was no indication that the gap between bigh and low illumination conditions would be appreciably better filled by this scheme, so that the construction of such an apparatus was not undertaken.

Some observations made with this clock-hand device, using white light ${ }^{*}$, indicated a bending back or return of the after-image just before its disappearance at the highest illumination. This might be due to a failure of the rectilinear logarithmic relation between diffusivity and illumination, such as some earlier work on critical frequency suggested, or again it might be due to the rod function showing a tendency to increase its diffusivity just before failure. The clock-hand observations differed in magnitude from the ones plotted, but in the opposite direction to what better fixation would have accounted for, probably due to the different part and area of retina used.

\section{Discussion.}

The lagging of a blue image after a red is such a striking sight that it seems unlikely to have escaped observation, although $\mathrm{I}$ can find no mention of it. As an effect predicted from a physical theory of the transmission of impressions from the retina to the seat of consciousness it may, I think, claim novelty.

The positive after-image has, of course, been known for a very long time, and it is, moreover, a part of von Kries's Duplicitäts-theorie that this image is the rod image, delayed in perception by the greater reaction time of the rods. The existence of a relationship between the illumination and the time-interval separating the after-image from the primary, such as is here suggested and in part borne out, appears to be new.

* When the white hands are illuminated by the yellow-white light of the tungsten lamp, the after-image appears blue; if a blue glass is placed over the eye the after-image appears brown, pointing clearly to the colour being a subjective contrast effect. 
Some of the interesting experiments described by Shelford Bidwell * would, with very slight modifications, have shown all the effects which form the subject-matter of this paper. One of his experiments, for instance, was the rotation of a spectrum about an axis beyond the red end. The different apparent positions of the various colours probab ly escaped him owing to their gradual transition one into another, while a crude artificial spectrum of several patches of colour would have revealed the peculiarity. The curved after-image which he shows following the spectrum I believe has that shape due to imperfect fixation, as illustrated in fig. 8, or perhaps to its extending over a sufficient extent of the retina to show a probable variation of diffusivity from centre to periphery.

One of Bidwell's diagrams, illustrating the fluctuating. intensity of the extended image of a rotating bright slit

Fig. 9.
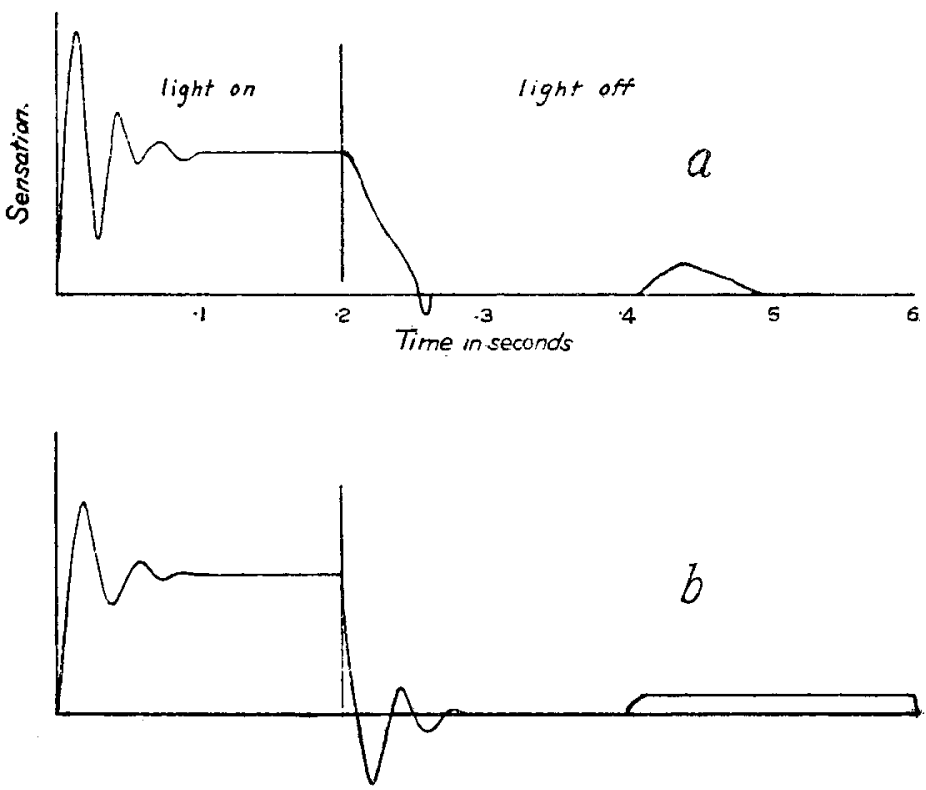

a. Progress of sensation with time due to $a: 1 / 5$ second exposure according to Bidwell : $b$, electrical transmission of brief stimulus by line containing inductance and capacity.

and its after-image, deserves attention in any discussion of the visual processes as in part phenomena of physical transmission. This diagram, produced in fig. $9 a$, is to be compared with fig. $9 b$, which is the record of a brief

* Bidwell, Proc. Roy. Soc. lvi. 1894, p. 132. 
Vapou Pressure of Zinc, Culmium, und Mercury.

contact as transmitted by in electrical transmission line containing inductance and capacity. The similarity is very striking, especially if we add the delayed record of a second line of high resistance, constituting the after-image.

The postulation of inductance and capacity for the transmitting media in vision is, of course, a considerable step in advance over the simple type of diffusivity which is sufficient to handle the brief exposure and intermittent phenomena. The oscillatory character of the image as described by Bidwell may just as well be ascribed to an oscillatory photo-chemical reaction, of which several are known. At the same time the very great light thrown on these transient visual effects by treating them as physical transmission phenomena will, I think, entitle visual "diffusivity" to a place of some importance in future theories of vision.

Physical I,aboratory,

The United Gas Improvement Co., Philadelphia, Pa.

III. The Vapontr Pressure of Zinc, Cadmium, and Mercury. By. A. C. Egerton *

$\mathrm{N}$ a quest for a method to determine the vapour pressures 1 of various solid elements, it was attempted to employ a process similar to that which has been used by L. Pfaundler (Ann.d. Phys. (3) lxiii. p. 36, 1897) and others, but the results did not promise to lead to a satisfactory general method. Subsequently Professor Nernst drew my attention to Knudsen's $\dagger$ work on the vapour pressure of mercury, and it has beon found possible to adapt his method to the determination of the vapour pressures of other elements.

The following is an account of the determinations of the vapour pressure of zine, cadmium, and mercury. It is hoped to continue the investigations for other elements and, by comparing the results obtained for different elements, to gain a measure of the forces between the atoms. The results for these elements already show that that hope is well founded. In 1909 Knudsen published an account of the determination of the vapour pressure of mercury by a method which depended on his investigations of the kinetic flow of molecules through small apertures and tubes. The apparatus consisted of a glass tube, separated into two compartments by a thin

* Communicated by Prof. H. L. Callendar, F.R.S.

† Ann. d. Plyys. (4) xxix. j. 179 (1909).

Phil. Mag. S. 6. Vol. 33. No. 193. Jan. 1917. 